\title{
The Nature, Origin and Propagation of the Electric Field: A New Insight to Fundamental Physics
}

\author{
Narahari V. Joshi \\ Department of Physics, University of Los Andes, Merida, Venezuela \\ Email: joshi@ula.ve
}

Received 25 April 2016; accepted 16 June 2016; published 21 June 2016

Copyright ( 2016 by author and Scientific Research Publishing Inc.

This work is licensed under the Creative Commons Attribution International License (CC BY).

http://creativecommons.org/licenses/by/4.0/

cC) (i) Open Access

\begin{abstract}
The nature, origin and propagation of the electric field are discussed for the first time on the basis of the presence of vibrating strings in the space and their self-excitation process. It is considered that the electron is formed from strings and it has specific vibrational frequency. This excites the strings which are close by with the self-excitation process. This procedure which is continuous in the space according to the symmetry and vibrational energy in the form of waves spreads near the electron (or the charge particle), which behaves and carries energy known as electric field. In fact, the electron does not continuously emit energy in any form but induces (or excites) and organizes energy in a self-sustain vibrational form and extends in three dimensional space. Only on the basis of the presence of strings (vibrational energy), several electromagnetic phenomena have been explained in a consistent way. The vibrational nature of the electric field is also examined with the help of Stark effect and X-ray diffraction approach to support the present view.
\end{abstract}

\section{Keywords}

Electric Field, Strings as Compact Liquid, Self Excitation

\section{Introduction}

In spite of several years of research work, important aspects of electric field are not well understood, particularly its nature and how it propagates in vacuum. According to the accepted theory [1] a charge particle like the electron emits energy continuously in some form. Following laws of symmetry, it is propagated in a spherical shape. At a time $t$ the sphere of radius ct is filled with electric energy where $c$ is the velocity of light. After time dt the sphere of radius $c(t+d t)$ is filled with energy. The most important aspect is that the intensity of the field within 
the radius ct is not altered and extra energy or field is observed in the outer shell of thickness cdt. It is worth mentioning that during this process the mass of the electron is the same. This means that energy is not conserved and the electron or the charge particle cannot emit energy (or field) continuously.

This observation has no explanation on the basis of classical electrodynamics. However, in the frame work of Quantum Field Theory (QFT) some explanation is provided. According to it (QFT), there exist intermediate energy states. The sum of these states forms an integral which includes all energy states and momentum. Integrals related with momentum become divergent. The renormalization route converts them into virtual (off shell) particles. This results in absorption and emission of virtual particles by the electron (ultraviolet divergence). This hypothesis based on QFT theory helps to explain the conservation of the charge of the electron [2].

A virtual particle is an explanatory conceptual entity that is originated in mathematical calculations in quantum field theory and it is a confusing subject. This is a highly speculative assumption and the absorption of the virtual particle is neither conformed theoretically nor experimentally. Sometimes, it is considered as disturbances in the field. Meanwhile, the absorption of the field in vacuum by vibrating strings and its feedback process given by Van der Pol nonlinear differential equation do explain satisfactorily the conservation of charge and mass [3].

The other very significant and fundamental aspect is that the charge particle (positive or negative) forms equivi-potential circles (or points) and a field of lines (or sometimes field of force) are directed from the positive charge outwards in the space meanwhile for the negative charge the field of lines are attracted towards it [1]. This explains the charge distribution in three dimensional space and the force can be calculated at any point. However, it is never discussed why the field is directed towards the negative charge and it originates from the positive charge. Meanwhile, the equi-potential surfaces have exactly the same properties. This contradiction, which forms the base for electromagnetism, is completely overlooked. Recently, this paradox has been discussed due to the motion of organized vibrating strings which not only explains the detail mechanism of formation of equi-potential circles but also elucidates why they have clock wise and anti-clock wise properties [3] [4]. Moreover, it provides information about how and why the forces of attraction and repulsion are originated in between two charge particles i.e. force at a distance [4].

Recently, some work has been carried out in this direction and Gauss law has been re-examined and it is established that the energy does absorb in vacuum and space is filled with an excitable medium [5]. This is also confirmed by Casimir effect [6] which is associated with zero point energy of quantized field. The direct experimental confirmation for the presence of energy in vacuum has been investigated and several experimental data and patents have been reported [7]-[9]. Important aspect is that vacuum energy can be converted into electromagnetic energy and not any other form. According to the quantum field theory also, the field can be visualized assuming that the space is filled with interconnecting vibrating units similar to strings.

A re-examination of Gauss law [5] also shows that the absorption of energy is roughly proportional to the inverse of the square of the distance from the electron, indicating that a considerable energy is absorbed very close to the electron. This has a vibrational nature and it partly provides a feed back to the vibrational energy of the electron [3] [5] and in some way gives pressure on the electron which can be associated with the Poincare stress. In fact, the details and the origin for the stress has not received due attention [1]. However, the absorption of energy by strings very close to the electron does provide a possible explanation.

Moreover, the above point of view, namely the considerable absorption of vibrational energy associated with strings very close to the electron does explain the cloudy nature around the electron. The density of the cloud varies from point to point very close to the electron and this cloud moves with the electron. A careful measurement shows that the intensity of the electric field is slightly altered because of the shielding effect of clouds. In fact, the distribution of charge of the electron can be written as

$$
\alpha_{\text {observed }}=\alpha_{\text {naked }}+\alpha_{\text {cloud }}
$$

where $\alpha_{\text {observed }}$ is the charge of the electron that is observed $\left(1.6 \times 10^{-19}\right.$ Coulombs); $\alpha_{\text {naked }}$ is the true charge which is not measured and $\alpha_{\text {cloud }}$ is the charge distribution around the central part. According to the quantum mechanical model, at the central part of the electron the cloud density is maximum, while it reduces considerably outside.

This origin for the presence of clouds near the electron has never been examined critically. However, in the frame work of quantum field theory, it is explained on the basis of ultra-violet divergence of fields by using renormalization process. According to it, the contribution in the divergent integrals associated with the correction 
term to the mass or charge of the particle. However, the most important aspect namely the origin for $\alpha_{\text {cloud }}$, the correction term to the cloud of electron, is not addressed in QFT [2]. It is worth mentioning that the point of view expressed above indicates that the origin for clouds lies in the vibrational energy very close to the electron absorbed by the mechanism mentioned earlier [5].

Recently, several fundamental aspects of electromagnetic fields such as Maxwell's equations [10], Lorentz force [11] electrostatic attraction or repulsion [4] etc. have been re-examined on the basis of strings as a compact incompressible liquid. According to the string theory the space is filled with small vibrating units of the order of Planck's length $\left(10^{-33} \mathrm{~cm}\right)$. Several interesting aspects of the electric and magnetic fields are explained with branes, which are a physical entity that generalizes a point particle to higher dimensions. $\mathrm{D}_{1}$ brane is like a string, it vibrates and it also has quantum fluctuations. Several properties related with elementary particles have been explained with the extra dimensions of branes. According to the string theory, the D-brane plays a crucial role. It is assumed that it carries the electric or magnetic field and open strings couple to the electromagnetic field at their end points. Maxwell fields are associated with the attachment of the open string and D-brane (in an extra dimension). Even though a considerable work has been reported in this direction, a totally different approach is taken in the present work. It is worth mentioning that in this discussion only the presence of strings in the vacuum is taken into account as vibrating units and no other aspects of the string theory such as the presence of branes of any dimension ( $\mathrm{D}_{0}$ (point), $\mathrm{D}_{1}$ (line), $\mathrm{D}_{2}$ (surface) and so on) are considered. Quantum harmonic oscillators (or vibrating units) in space permit us to consider conversion of energy into kinetic, potential and also in storage form. The direct consequence of it is that the space becomes an excitable medium through which the energy in some form can be transported (not by flow, motion or stream of any kind) by a non-conventional mechanism like propagation of induced "self-excited vibrations" in strings.

The other significant aspect of string theory is that particles are not point like. Instead they are vibrational modes. This means that elementary particles are formed from a specific arrangement of vibrating strings and therefore all particles have vibrating nature with specific frequency. In addition to this, the electron has a rotational motion (or spin). By considering the vibrational and rotational motions of electron, several properties of electromagnetism have been coherently explained earlier just by taking into account strings in a compact form of dry liquid [3]-[5] [10] [11]. However, it differs from the conventional liquid as every element of it (vibrating string) has significant amount of energy in a storage form which is convertible into kinetic and potential energy. As mentioned earlier because of the feedback mechanism based on Van der Pol equation, the energy is conserved due to self-sustained vibrations. This includes the conservation of the charge and mass (both structural and electromagnetic) of the electron and details have been discussed extensively earlier by Joshi [3].

\section{Self-Excited Vibrations and Propagation}

In fact, as mentioned earlier, the electron does not emit energy or field. The vibrations associated with electrons induce excitations in vibrating strings with which the space is filled. A self-excitation process [12]-[14] of vibrational energy advances in 3-D space and gives the impression that the electron or charge particle is emitting continuously energy or electric field in the space.

Self- excited systems begin to vibrate with their own accord under special conditions [12]. The energy required for these vibrations is obtained from a uniform source associated with the system itself (in the present case, vibrational energy of strings) and there is some inherent mechanism which supplies energy to start and maintain harmonic oscillations with certain frequency. If the system is disturbed for any reason the forces are created and they may lead the system away from the equilibrium position and then it will start oscillations provided that the conditions are suitable. When the cause for self-oscillations stops, the forces related with it disappear.

It is known from string theory that strings behave like quantum harmonic oscillators with quantum fluctuations (zero point energy) [15] and form a self-excitable system. The self-excitation process can be originated from several mechanisms but the most obvious in the present system is due to "Stick slip" process according to which one surface slides on another with dry friction [13]. This process is also known as friction induced self-excited vibration. Earlier investigation reveals that this is more probable when vibrating elements are very small as it is the present case. Favorable conditions for this type of excitation are that the surface should be in close contact for some time and then move with a low velocity. These conditions are completely fulfilled in strings as a compact form of liquid. Self-excitation can also take place due to flow induce vibrations in a com- 
pact liquid of strings [12] [13]. Both processes are obvious and effective which help the vibrations of electrons to excite and organize scheme of strings.

Now, let us imagine a vibrating electron (or charge particle) in three dimensional space which will induce self-activated vibrational energy in the nearest region. According to the symmetry, the nearest region will activate vibrations in strings which lie in the next circle and so on. After time t, the strings in radius ct will have vibration energy due to the self-activation process. However, it is worth to mention that the amplitude of vibrations (or the intensity) will go on reducing from one stage to another as the number of participating layers of strings increases [3]. Thus, the electron or the charge particle does not emit energy in any form but the vibrations associated to it induce self-activated vibrations in the surrounding system which in turn propagates in three dimensional space. This means that the field energy is directly associated with the vibrational energy.

\section{Interaction of the Electric Field with Vibrating Systems}

To appreciate the nature of the electric field and its association with vibrations, it is necessary to examine the interaction of the electric field with vibrating systems as it is difficult to estimate or evaluate the properties directly because of unknown parameters of strings related with vibrations and quantum harmonic oscillators.

According to the string theory also, some of the properties of strings are associated with quantum harmonic oscillators [15]. Because of their compact nature, it can be assumed that they form a system of strongly coupled harmonic oscillators.

Morse potential is an interatomic interaction model between two atoms or molecules. It is better approximated to the potential associated with the vibrational nature of the system [13] [14]. Obviously, it interacts strongly with other vibrating systems like the electric field. An important characteristic feature of strong coupling interaction is the splitting of the energy levels and it is frequently associated with the perturbation in Morse potential, as in strong coupling the potential energy interacts with each other. The effects of the external electric field on Morse potential have been investigated earlier [13] [14] and an analytical formulation had been developed which explains successfully the frequency and the bond length dependence for diatomic molecules.

Delly [14] added an extra term in Morse potential

$$
\begin{gathered}
V(r)=\operatorname{De}(1-\eta)^{2}+f(E) \cdot r \\
\eta=\mathrm{e}^{-a\left(r-r_{o}\right)}
\end{gathered}
$$

where $f(E)$ is the perturbation induced by the applied electric field. Here $r$ is the distance between two nucleus of interest and $r_{o}$ is the equilibrium distance. Obviously " $a$ " has dimensions of (length) ${ }^{-1}$. De is the energy associated with the dissociation of the bond length. Taking into account the additional term, the frequency and the bond length have been examined for some molecules like Nacl, $\mathrm{H}_{2} \mathrm{O}$ and experimental data are in agreement with the calculated values. The addition of the term $f(E) \cdot r$ in the potential of the harmonic oscillator also implies that the energy associated with the electric field is related with the vibrational energy. This approach has been further extended by Hashjin and Mott [13].

The detailed calculations of energy splitting have been carried out earlier by Novotny [16] by considering the equation of motion for a coupled system in which the constant of coupling can be varied and its effect on energy splitting is clearly determined experimentally. In the adiabatic limit, it is possible to transfer energy from one oscillator to the other through resonance [14] [16]. Therefore, it is necessary to examine the effect of the electric field on oscillating systems such as coupled quantum dots or a system of atoms and molecules in the external electric field. This means Stark effect.

\section{Vibrational Stark Effect (VSE)}

It is known that an electric field, internal or externally applied, perturbs molecular vibrations considerably. The changes induced by the applied electric field are measured by VSE, thus it provides a direct mapping between vibrational modes and electric field.

One of the few experimental evidences where the electric field is directly related with the vibrational energy of the system at atomic or molecular level is Stark effect; which shows the shifting and splitting of energy states due to the presence of the external electric field. VSE provides a direct relation between the observed variation in the vibrational modes and the applied electric field. Recently, it has been suggested that the external electric 
field alters the potential energy of the surface perturbing the Morse potential of the molecule. This causes the change in the bond length and hence changes in the frequency. The field induced changes in the frequency and the force constant are given by [13].

$$
\begin{aligned}
d v & =v_{E}-v_{0} \\
d k & =k_{E}-k_{0}
\end{aligned}
$$

where the subscript $E$ shows the value perturbed by steady state electric field and subscript o denotes a free field value.

A detailed calculation has been carried out for the applied electric field and the field induced frequency is given [13]

$$
V_{E}=K v_{0}
$$

where $K$ is a constant and whose value depends upon the magnitude and direction of the applied electric field. The obtained results are compared with the calculated values for several molecules like $\mathrm{H}_{2}, \mathrm{~N}_{2}, \mathrm{O}_{2}$ and $\mathrm{F}_{2}$ (homo-nuclear ) and $\mathrm{HF}, \mathrm{HCl}, \mathrm{CO}$ (hetero-nuclear) and they are in agreement .The details are given by Hashjin and Matt [13]. It is worth to point out that the theory is tested for strongly covalent bonds which are described by harmonic oscillators and whose energy states lie at the bottom of the Morse potential well.

The additional effect is also observed by X-ray diffraction technique where the electric field induces the changes in the bond length or inter- atomic distances [17]. The above studies reveal that electric field, which is associated with vibrational motion, interacts with the oscillating system of the atoms and molecules. The above mentioned experimental techniques strongly support the vibrational nature of the electric field. This might help to understand the conversion of field into particles (photons) or vice versa as the energy of both depends upon the frequency. This is mathematically explained with the help of the creation and annihilation operatorsin QFT.

The electron and its associated field have vibrational nature. Therefore, it is interesting to examine other elementary particles and the nature of the corresponding fields. In a compact liquid, as considered in the present case, the mechanism associated with Van der Pol equation is not valid. However, a liquid in a confined region like the nucleus has several particles and their interacting fields which give rise to the damping coefficient and increase the magnitude of the constant of string $\mu$. Van der Pol equation, therefore, might play a crucial role in the system which is given by [3]

$$
\frac{\mathrm{d}^{2} x}{\mathrm{~d} t^{2}}+\zeta\left(1-x^{2}\right) \frac{\mathrm{d} x}{\mathrm{~d} t}+\mu x=0
$$

Here $\zeta$ is a damping coefficient. Recently this equation has been solved by the algebraic method and it is found that constants $\zeta$ and $\mu$ play a very crucial role. The solution of the above equation is given [18]

$$
x=\mathrm{e}^{-\zeta t}[b \sin (\omega t+\phi)]
$$

where $b$ and $\phi$ are constants which depend upon the initial conditions. A detailed analysis reveals that the frequency mainly depends upon the value of $\mu$ meanwhile $\zeta$ determines the exponential fall. The value of $\mu$ is related with the potential energy of the oscillator which becomes considerably high in the presence of particles and their interactions. Naturally fields have high frequency. Obviously, for higher values of $\zeta$ (damping coefficient), the forces have very short range. A further systematic investigation with this approach might provide information about the range of the force and their interactions. If so, the presence of vibrating strings in the form of a compact liquid might be a step ahead in the unification process.

\section{Conclusion}

The nature, origin and details of the propagation of the electric field on the basis of self-excitation process are discussed. Contrary to the accepted theories, it is found that the electron induced vibrational energy by self-excitation mechanism. The excitation process continues in space and the vibrational energy in the form of electric field spreads. Unexplained properties like electron cloud and Poincare stress become the natural consequences of the proposed theory. The electric field is examined with the help of VSE and X ray diffraction approaches. It is worth mentioning that several electromagnetic properties have been explained in a consistent manner by assuming only the presence of vibrating strings in the space. 


\section{References}

[1] Feynman, R.P., Leighton, R.B.M. and Sands, M. (1969) The Feynman Lectures on Physics, Vol. 2, Chapter 4. Addision-Wesley, Boston.

[2] Bogolyubov, N.N. and Shirkov, D.V. (1982) Introduction to the Theory of Quantized Fields, Chapter 3. Benjamin, Reading.

[3] Joshi, N.V. (2013) Physics Essays, 26, 61-67.

[4] Joshi, N.V. (2013) World Journal of Mechanics, 3, 307-309. http://dx.doi.org/10.4236/wjm.2013.37032

[5] Joshi, N.V. (2015) Journal of Modern Physics, 6, 2035-2039.

[6] Milonni, P. (1994) The Quantum Vacuum. An Introduction to Quantum Electro-Dynamics, Chapter 1. Academic Press, San Diego.

[7] Bearden, T.E. (2004) Energy from the Vacuum, Chapter 7. Cheniere Press, Santa Barbara.

[8] Haisch, B. and Moddel, G. (2008) Quantun Vacuum Energy Extraction. US. Patent No. 7379286.

[9] Maclay, G., Hammer, J.R., Clark, G.M., Kim, Y. and Kir, A. (2004) Study of Vacuum Energy for Breakthrough Propulsion. NASA Report OR-213311.

[10] Joshi, N.V. (2015) Journal of Modern Physics, 6, 921-926. http://dx.doi.org/10.4236/jmp.2015.67096

[11] Joshi, N.V. (2014) World Journal of Mechanics, 4, 247-250.

[12] D’Souza, A.F. and Dweib, A.H. (1990) Journal of Sound and Vibration, 137, 177-190. http://dx.doi.org/10.1016/0022-460X(90)90787-Z

[13] Hashjin, S.S. and Matt, C.F. (2013) Journal of Chemical Physics, 139, 144101-144112. http://dx.doi.org/10.1063/1.4820487

[14] Delley, B. (1998) Journal of Molecular Structure: Theochem, 434, 229-237.

[15] Zwiebach, B.A. (2009) First Course in String-Theory, Chapter 12. Cambridge University Press, Cambridge. http://dx.doi.org/10.1017/CBO9780511841620

[16] Novotny, L. (2010) American Journal of Physics, 78, 1199-1202. http://dx.doi.org/10.1119/1.3471177

[17] Semen, V.G., Vladimir, G.T. and Ullrich, P. (2005) Acta Crystallographica Section A, 61, 387-396.

[18] Akbari, M.R., Ganji, D.D., Mujidian, A. and Ahmadi, A.R. (2014) Frontiers of Mechanical Engineering, 9, 177-190. http://dx.doi.org/10.1007/s11465-014-0288-8

\section{Submit or recommend next manuscript to SCIRP and we will provide best service for you:}

Accepting pre-submission inquiries through Email, Facebook, Linkedin, Twitter, etc A wide selection of journals (inclusive of 9 subjects, more than 200 journals)

Providing a 24-hour high-quality service

User-friendly online submission system

Fair and swift peer-review system

Efficient typesetting and proofreading procedure

Display of the result of downloads and visits, as well as the number of cited articles

Maximum dissemination of your research work

Submit your manuscript at: http://papersubmission.scirp.org/ 